\title{
Primitive Spatial Graphs and Graph Minors
}

\author{
Makoto Ozawa and Yukihiro TsUTSUMı \\ Department of Natural Sciences \\ Faculty of Arts and Sciences \\ Komazawa University \\ 1-23-1 Komazawa, Setagaya-ku \\ Tokyo 154-8525 - Japan \\ w3c@komazawa-u.ac.jp \\ Department of Mathematics \\ Faculty of Science and Technology \\ Sophia University \\ Kioicho 7-1, Chiyoda-ku \\ Tokyo 102-8554 - Japan \\ tsutsumi@mm. sophia.ac.jp
}

Received: April 10, 2006

Accepted: February 6, 2007

\begin{abstract}
Robertson, Seymour, and Thomas characterized linkless embeddings of graphs by flat embeddings, and determined the obstruction set for linkless embeddings. In this paper, we extend flat embeddings to "primitive embeddings" as linkless embeddings to knotless embeddings. Although the obstruction set for knotless embeddings has not been determined, fundamental theorems and conjectures are obtained.

Key words: spatial graph, graph minor.

2000 Mathematics Subject Classification: primary 57M25, 57M15; secondary 05C83, $05 \mathrm{C} 10$.
\end{abstract}

\section{Introduction}

Let $G$ be a finite graph. An embedding of $G$ into the 3 -sphere $S^{3}$ is said to be knotless (resp. linkless) if it contains no non-trivial knot (resp. non-trivial link) from cycles of $G$. Let us say that an embedding $\phi$ of $G$ in $S^{3}$ is free if the fundamental group of $S^{3}-\phi(G)$ is free. An embedding $\phi$ of $G$ in $S^{3}$ is said to be flat if for every cycle $C$ of $G$, there exists a disk in $S^{3}$ internally disjoint from $\phi(G)$ whose boundary is $\phi(C)$. The Petersen family is the set of all graphs that are obtained from $K_{6}$ or $K_{3,3,1}$ by a finite sequence of $\Delta \mathrm{Y}$-exchanges. We remark that the Petersen family results in the

The second author was partially supported by Research Fellowships of the Japan Society for the Promotion of Science for Young Scientists. 
set of all graphs that can be obtained from $K_{6}$ by means of $Y \Delta$ - and $\Delta Y$-exchanges. See figure 5 in section 2 for $Y \Delta$ - and $\Delta Y$-exchanges and [15, figure 1] for the Petersen family. A graph is a minor of another if the first can be obtained from a subgraph of the second by contracting edges. Robertson, Seymour, and Thomas ([13]) proved that the followings are equivalent.

(i) $G$ has an embedding whose every subgraph is free,

(ii) $G$ has a flat embedding,

(iii) $G$ has a linkless embedding,

(iv) $G$ has no minor in the Petersen family.

\subsection{Fundamental Theorem and Conjecture}

For knotless embeddings, such characterization has yet to be done. Here, we introduce a notion of primitive embeddings, which will make a contribution to the knotless embedding problem. An embedding $\phi$ of a graph $G$ in $S^{3}$ is said to be primitive if for each component $G_{i}$ of $G$ and any spanning tree $T_{i}$ of $G_{i}$, the bouquet $\phi\left(G_{i}\right) / \phi\left(T_{i}\right)$ obtained from $\phi\left(G_{i}\right)$ by contracting all edges of $\phi\left(T_{i}\right)$ in $S^{3}$ is trivial.

Theorem 1.1. An embedding $\phi$ of a graph $G$ in $S^{3}$ is primitive if and only if the restriction $\left.\phi\right|_{G^{\prime}}$ is free for all connected subgraph $G^{\prime}$ of $G$.

Therefore, a flat embedding is primitive, but the converse is not true. For example, a non-split spatial graph that consists of trivial disjoint cycles is primitive, but not flat. Besides, a primitive embedding is knotless since the fundamental group of a nontrivial knot complement is not free. As we know afterward, the notion of primitive embedding is closely related to knotless embedding. We expect:

Conjecture 1.2. A graph has a primitive embedding if and only if it has a knotless embedding.

Remark 1.3. Minimally knotted embeddings of a connected graph are knotless, but not primitive since itself is not free by [11].

The following theorem assures us of a relation about edge-deletions and contractions (compare with [16, (1.8)] and an alternate version of 7.5 in [18]).

Theorem 1.4. Let $\phi$ be an embedding of a graph $G$, and $e$ be an edge of $G$ with distinct end vertices. Then $\phi$ is primitive (resp. knotless) if and only if both of $\left.\phi\right|_{G-e}$ and $\left.\phi\right|_{G / e}$ are primitive (resp. knotless).

For a $\Delta Y / Y \Delta$-exchange, we have a relation between two embeddings (compare with the Lemma in [10]). 
Theorem 1.5. Let $H$ be a graph with a 3-cycle $C$, and $\phi^{\prime}$ be an embedding of $H$ such that $C$ bounds a disk $D$ internally disjoint from $\phi^{\prime}(H)$. Let $G$ be a graph obtained from $H$ by a $\Delta Y$-exchange, and $\phi$ be an embedding of $G$ which is obtained from $\phi^{\prime}$ by a $\Delta Y$-exchange on $D$. Then $\phi$ is primitive (resp. knotless) if and only if $\phi^{\prime}$ is primitive (resp. knotless).

\subsection{Graph minor}

For graph minors, primitive embeddings are similar to knotless embeddings. An abstract graph is primitive if it has a primitive embedding.

Theorem 1.6. The property of having a primitive embedding is preserved under taking minors.

Let $\mathcal{C}$ be a property closed under minor-reduction. The obstruction set for $\mathcal{C}$, denoted by $\Omega(\mathcal{C})$, is the set of all minor-minimal graphs which do not have $\mathcal{C}$. It is well-known that $\Omega(\mathcal{C})$ is finite $([12])$. Therefore, it is a characterization for a property $\mathcal{C}$ to determine the obstruction set. Let $\mathcal{K} \mathcal{L}$ be the property that a graph has a knotless embedding. Kohara and Suzuki ([10]) conjectured that $\Omega(\mathcal{K} \mathcal{L})$ is equal to the union of $K_{7}$-family and $K_{3,3,1,1}$-family, where $K_{7}$-family (resp. $K_{3,3,1,1}$-family) is the set of graphs that are obtained from $K_{7}$ (resp. $K_{3,3,1,1}$ ) by $\Delta Y$-exchanges. Recently, Foisy ([4]) discovered a new intrinsically knotted graph, which we call Foisy graph and denote by $F$, belongs to $\Omega(\mathcal{K} \mathcal{L})$, but is independent of the $K_{7^{-}}$and $K_{3,3,1,1}$-family. Let $\mathcal{P}$ be the property that a graph has a primitive embedding.

Theorem 1.7. The $K_{7}$-family and $K_{3,3,1,1}$-family are contained in the obstruction set $\Omega(\mathcal{P})$ for primitive graphs.

Remark 1.8. Since the Foisy graph $F$ is not primitive, there exists a graph $G \in \Omega(P)$ which is a minor of $F$. This graph $G$ will be a new element of $\Omega(P)$ other than $K_{7}$ and $K_{3,3,1,1}$-family, since $F$ has no minor in $K_{7}$ nor $K_{3,3,1,1}$-family.

It is clear that a graph obtained from a planar graph joined with two vertices has a knotless embedding. Indeed, when we construct an embedding of the graph forming a plane graph joined with the North Pole and the South Pole, any cycle is a bridge number one knot, hence unknotted.

Theorem 1.9. A planar graph joined with two vertices is primitive.

Remark 1.10. For any graph $G$ in $K_{7^{-}}$or $K_{3,3,1,1}$-family, any minor of $G$ forms a planar graph joined with two vertices. However, there is an edge $e$ of the Foisy graph $F$ such that $F-e$ is not a form of planar graph joined with two vertices.

\subsection{Primitive embedding}

By Theorem 1.1 and the Robertson-Seymour-Thomas theorem, flat embeddings are primitve. Conversely, primitive embeddings are flat if we restrict on the structure of graphs. 


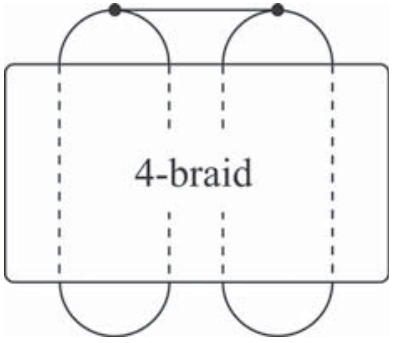

(a) $H_{1}$

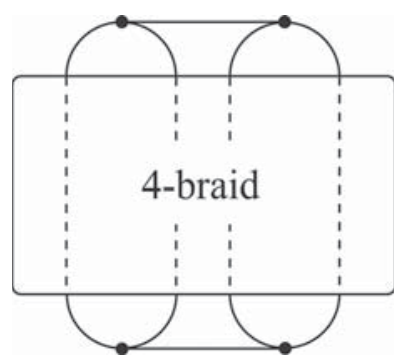

(b) $\mathrm{H}_{2}$

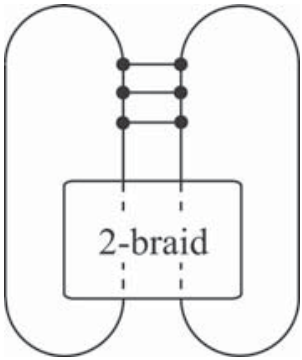

(c) $H_{3}$

Figure 1 - Primitive embedding of $H_{n}(n=1,2,3)$

Theorem 1.11. If a graph has no disjoint cycles, then any primitive embedding of the graph is also flat.

If a graph has disjoint cycles, then primitive embeddings of the graph are not flat generally. We characterized primitive embeddings of a "handcuff graph with $n$-bridges" $H_{n}$ for $n=1,2,3$.

A link $L$ is called a 2-bridge link if there is a sphere which intersects $L$ transversely in four points and decomposes $\left(S^{3}, L\right)$ into two trivial 2-string tangles. A link $L$ is called a $(p, q)$-torus link if there is a solid torus $V$ standardly embedded in $S^{3}$ so that $L$ is contained in $\partial V$ as a $(p, q)$-curve, where $(0,1)$ and $(1,0)$ correspond to a meridian and a preferred longitude for $V$ respectively. An unknotting tunnel $\tau$ is an arc such that $\tau \cap L=\partial \tau$ and $S^{3}-\operatorname{int} N(L \cup \tau)$ is a handlebody. We note that every 2-bridge link and torus link admits an unknotting tunnel.

We recall that a complete classification of the unknotting tunnels for 2-bridge links is given by Adams and and Reid ([1]), and that only the upper and lower tunnels are unknotting tunnels. See [8] for the classification of unknotting tunnels of 2-bridge knots.

Theorem 1.12. Any primitive embedding of $H_{n}(n=1,2,3)$ forms :

(i) a 2-bridge link with an upper tunnel if $n=1$.

(ii) a 2-bridge link with an upper tunnel and a lower tunnel if $n=2$.

(iii) $a(2, q)$-torus link with three parallel tunnels if $n=3$.

From Theorem 1.12, we know that primitive embeddings are "rigid" as the connectivity of the graph becomes higher. It should be noted that any link contained in any primitive embedding of $K_{6}$ is a $(2, q)$-torus link since $K_{6}$ contains $H_{3}$ as a primitive embedding by Theorem 1.4. In this direction, we present a non-planar graph which has exactly two primitive embeddings up to reflection, as follows. 


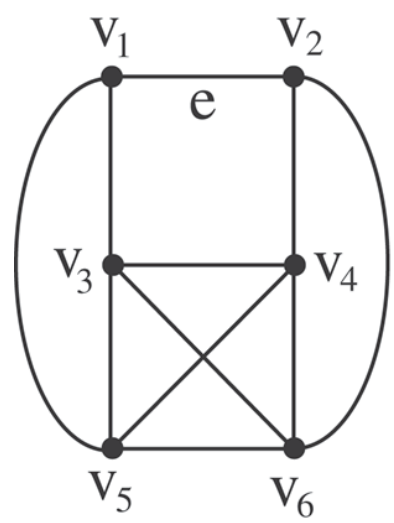

(a) $K_{5}^{\prime}$

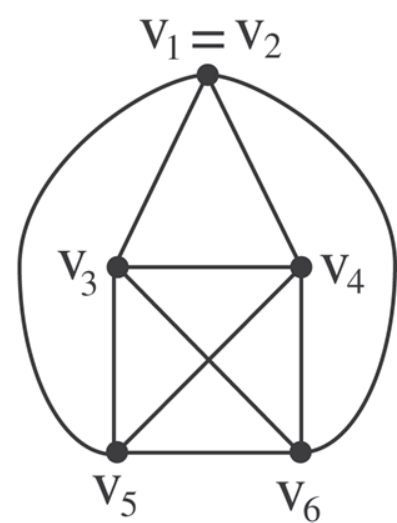

(b) $K_{5}$

Figure $2-K_{5}^{\prime}$ and $K_{5}$

Let $K_{5}^{\prime}$ be a graph obtained from $K_{5}$ by once de-contracting, that is, there exists an edge $e$ of $K_{5}^{\prime}$ such that $K_{5}^{\prime} / e$ is isomorphic to $K_{5}$. See figure 2 .

Lemma 1.13. $K_{5}^{\prime}$ has exactly two primitive embeddings up to reflection, as illustrated in figure 3.

Remark 1.14. It follows from Lemma 1.13 that the only pair of disjoint cycles $v_{1} v_{3} v_{5} v_{1}$ and $v_{2} v_{4} v_{6} v_{2}$ of $K_{5}^{\prime}$ forms the trivial link or the Hopf link in any primitive embedding of $K_{5}^{\prime}$.

By using Lemma 1.13, we can show a strong restriction on sublinks contained in a primitive embedding of a graph in the Petersen family.

Theorem 1.15. Any link contained in a primitive embedding of a graph in the Petersen family is either the trivial link or the Hopf link.

Generally, a primitive embedding places restrictions on its sublinks.

Theorem 1.16. An n-component link contained in a primitive embedding of a connected graph has bridge number $n$.

According to $[16,(1.7)]$, flat embeddings of a 4-connected graph are unique up to reflections. We also expect some rigidity of primitive embeddings.

Conjecture 1.17. Primitive embeddings of a 5-connected graph are unique up to reflections.

For planar graphs, by Theorem 1.11, any primitive embedding of a planar graph is flat if it has no disjoint cycles, hence primitive embeddings of such graphs are unique. This is the only case for planar graphs to have a unique primitive embedding. 


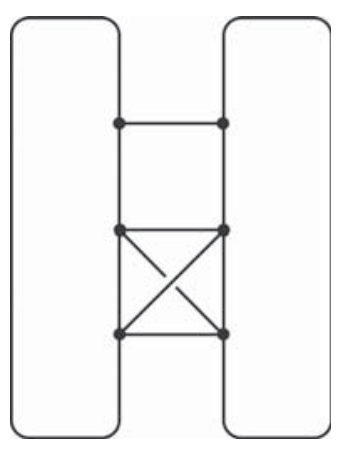

(a)

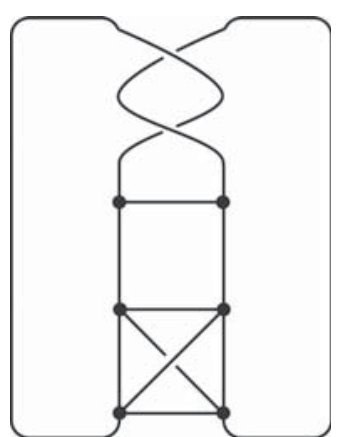

(b)

Figure 3 - two primitive embeddings of $K_{5}^{\prime}$

Theorem 1.18. A planar graph has a unique primitive embedding if and only if it has no disjoint cycles. Moreover, if a planar graph has disjoint cycles, then it has infinitely many primitive embeddings.

\section{Proof of Fundamental Theorems}

We refer the reader to $[6,7,17]$ for standard terminology in knot theory and threedimensional topology. A tangle is a pair of a 3-ball $B$ and properly embedded 1manifold $t$. When $t$ consists of $n$ arcs, we call the tangle an $n$-string tangle. An $n$-string tangle is said to be trivial if there are mutually disjoint $n$ disks $D_{i}$ 's in $B$ such that $\partial B \cap D_{i}$ is a subarc of $\partial D_{i}, D_{i} \cap t=t_{i}$, and $\partial D_{i}=t_{i} \cup(B \cap)-\partial B \cap D_{i}$, where $t_{i}$ is a component of $t$.

Let $\phi: G \rightarrow S^{3}$ be an embedding of $G$, and $e$ an edge of $G$. A simple closed curve on $\partial N(\phi(G))$ is called a meridian for $\phi(e)$ if it bounds a disk $D$ in $N(\phi(G))$ such that $D \cap \phi(G)$ is a single transverse point, where $N(\phi(G))$ is a regular neighborhood of $\phi(G)$. We denote by $\phi(e)^{*}$ the meridian for $\phi(e)$, and call such a disk $D$ a meridian disk for $\phi(e)$. For a subgraph $G^{\prime}$ of $G$, there are disjoint meridian disks for the edges of $G^{\prime}$ and we denote by $\phi\left(G^{\prime}\right)^{*}$ the disjoint union of meridians for all edges of $G^{\prime}$.

If we contract $\phi(e)$ in $S^{3}$, then the resultant embedding of $G / e$, denoted by the same symbol $\phi$ or $\left.\phi\right|_{G / e}$, is well-defined as $\phi(G / e)=\phi(G) / \phi(e)$. Notice that $\left(N(\phi(G / e)), \phi(G / e)^{*}, S^{3}\right)$ is homeomorphic to $\left(N(\phi(G)), \phi(G)^{*}-\phi(e)^{*}, S^{3}\right)$.

Let $T$ be a spanning tree of a connected graph $G$. We call the set of the edges $\mathcal{E}_{T}=E(G)-E(T)$ a base edge system for $T$. A set of edges $\mathcal{E}$ of $G$ is called a base edge system if there is a spanning tree $T$ such that $E(G)-E(T)=\mathcal{E}$.

If we contract all edges of $\phi(T)$ in $S^{3}$, then the resultant embedding of $G / T$ is uniquely defined. We denote this embedding by the same symbol $\phi$. Thus we regard $\phi(G) / \phi(T)$ as $\phi(G / T)$. 


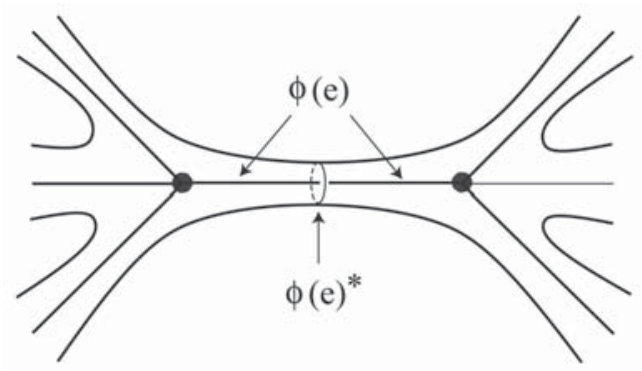

Figure $4-$ meridian $\phi(e)^{*}$ for $\phi(e)$

Lemma 2.1. An embedding $\phi$ of $G$ is primitive if and only if for any base edge system $\mathcal{E}=\left\{e_{1}, \ldots, e_{n}\right\}$ there exist disks $D_{1}, \ldots, D_{n}$ in $S^{3}-\operatorname{int} N(\phi(G))$ such that $\left|\partial D_{i} \cap \phi\left(e_{j}\right)^{*}\right|=\delta_{j}^{i}(i, j=1, \ldots, n)$.

Proof. Let $T$ be a spanning tree for $G$ and $\mathcal{E}_{T}=\left\{e_{1}, \ldots, e_{n}\right\}$ a base edge system for $T$. Put $B=S^{3}-\operatorname{int} N(\phi(T))$ and $B^{\prime}=S^{3}-\operatorname{int} N(\phi(T / T))$. Then, $\phi$ is primitive if and only if the $n$-string tangle $\left(B^{\prime}, \phi\left(e_{1} \cup \cdots \cup e_{n}\right) \cap B^{\prime}\right)$ is trivial. On the other hand, the latter condition of Lemma 2.1 holds if and only if the $n$-string tangle $\left(B, \phi\left(e_{1} \cup \cdots \cup e_{n}\right) \cap B\right)$ is trivial. Hence, Lemma 2.1 is proved since these two tangles are equivalent.

Remark 2.2. When $G$ is connected, $\phi: G \rightarrow S^{3}$ is primitive if and only if $S^{3}-$ int $N(\phi(G))$ is a handlebody and $\mathcal{E}_{T}^{*}$ is a primitive set on $\partial\left(S^{3}-\operatorname{int} N(\phi(G))\right)$ in the sense of Gordon [5] for any spanning tree $T$.

Proof (of Theorem 1.1). Suppose that an embedding $\phi$ of $G$ is primitive. Let $G^{\prime}$ be a connected subgraph of $G$ and $T^{\prime}$ a spanning tree for $G^{\prime}$. We extend $T^{\prime}$ to a spanning tree $T$ for $G$. Since $G$ is primitive, $\phi(G) / \phi(T)$ is a trivial bouquet. Therefore $\phi\left(G^{\prime}\right) / \phi\left(T^{\prime}\right) \subset \phi(G) / \phi(T)$ is also a trivial bouquet. Since $\pi_{1}\left(S^{3}-\phi\left(G^{\prime}\right)\right)$ is homomorphic to $\pi_{1}\left(S^{3}-\phi\left(G^{\prime}\right) / \phi\left(T^{\prime}\right)\right), \phi\left(G^{\prime}\right)$ is free.

Conversely, suppose that for any connected subgraph $G^{\prime}$ of $G, \phi\left(G^{\prime}\right)$ is free. Let $T$ be a spanning tree for $G$ and $\mathcal{E}$ the base edge system for $T$. Then $B=S^{3}-\operatorname{int} N(\phi(T))$ is a 3 -ball, and by the supposition, $B-\operatorname{int} N\left(\phi\left(\mathcal{E}^{\prime}\right)\right)$ is a handlebody for all subsets $\mathcal{E}^{\prime}$ of $\mathcal{E}$. By [5, Theorem 1] and Lemma 2.1, $\phi$ is primitive.

Proof (of Theorem 1.4). Primitive. Suppose that $\phi$ is primitive, and let $H$ be a connected subgraph of $G-e$. Then $\phi(H)$ is free since $H$ is a connected subgraph of $G$. Hence $\left.\phi\right|_{G-e}$ is primitive. Next, let $J$ be a connected subgraph of $G / e$, and $J^{\prime}$ be the corresponding subgraph of $G$. Then $\phi(J)$ is free since $\pi_{1}\left(S^{3}-\phi(J)\right) \cong \pi_{1}\left(S^{3}-\phi\left(J^{\prime}\right)\right)$. Hence $\left.\phi\right|_{G / e}$ is primitive. 
Conversely, suppose that both of $\left.\phi\right|_{G-e}$ and $\left.\phi\right|_{G / e}$ are primitive, and let $H$ be a connected subgraph of $G$. If $H$ contains $e$, then $\phi(H)$ is free since $\pi_{1}\left(S^{3}-\phi(H)\right) \cong$ $\pi_{1}\left(S^{3}-\phi(H / e)\right)$ and $\phi(H / e)$ is free. Otherwise, $\phi(H)$ is free since $H$ is a connected subgraph of $G-e$. Hence $\phi$ is primitive.

Knotless. Suppose that $\phi$ is knotless. If $\phi(G) / \phi(e)$ contains a non-trivial knot $K$, then $K$ passes through the vertex $\phi(e) / \phi(e)$ just once. Hence, $K$ is also contained in $\phi(G)$, and this contradicts that $\phi$ is knotless. It is easy to see that $\phi(G-e)$ is knotless since $G-e$ is a subgraph of $G$. Conversely, suppose that both of $\left.\phi\right|_{G-e}$ and $\left.\phi\right|_{G / e}$ are knotless, and that $\phi(G)$ contains a non-trivial knot $K$. If $K$ contains $e$, then it is contained in $\phi(G) / \phi(e)$. This contradicts that $\left.\phi\right|_{G / e}$ is knotless. Otherwise, $K$ is contained in $\phi(G-e)$, and we have a contradiciton in the same way. Hence, $\phi$ is knotless.

Before proving Theorem 1.5, we need some lemmas.

Lemma 2.3. Let $G$ be a graph with a valency 2 vertex $v$ to which two edges $e_{1}$ and $e_{2}$ incident, and $\phi$ be an embedding of $G$. Then $\phi$ is primitive (resp. knotless) if and only if $\left.\phi\right|_{G / e_{1}}$ is primitive (resp. knotless).

Proof. Suppose that $\phi$ is primitive. Then $\phi(G) / \phi\left(e_{1}\right)$ is primitive by Theorem 1.4.

Conversely, suppose that $\left.\phi\right|_{G / e_{1}}$ is primitive. By Theorem 1.4, $\left.\phi\right|_{\left(G-e_{1}\right) / e_{2}}$ is primitive since $\phi\left(G-e_{1}\right) / \phi\left(e_{2}\right)=\phi(G) / \phi\left(e_{1}\right)-\phi\left(e_{2}\right)$. And $\left.\phi\right|_{\left(G-e_{1}\right)-e_{2}}$ is primitive since $\phi\left(G-e_{1}\right)-\phi\left(e_{2}\right)=\left(\phi(G) / \phi\left(e_{1}\right)-\phi\left(e_{2}\right)\right) \cup($ isolated vertex $)$. Hence, by Theorem 1.4, $\left.\phi\right|_{G-e_{1}}$ is primitive. Therefore, $\left.\phi\right|_{G}$ is primitive.

Lemma 2.4. Let $G$ be a graph with a loop e, and $\phi$ be an embedding of $G$ such that $\phi(e)$ bounds a disk internally disjoint from $\phi(G)$. Then $\phi$ is primitive (resp. knotless) if and only if $\left.\phi\right|_{G-e}$ is primitive (resp. knotless).

Proof. Suppose that $\phi$ is primitive. Then $\phi(G-e)$ is primitive by Theorem 1.4.

Conversely, suppose that $\left.\phi\right|_{G-e}$ is primitive. Let $T$ be a spanning tree of $G$. Then $\phi(G) / \phi(T)$ is a trivial bouquet since $\phi(G) / \phi(T)=(\phi(G-e) / \phi(T)) \cup$ (trivial loop). Hence $\phi$ is primitive.

Lemma 2.5. Let $G$ be a graph with multi-edges $e_{1}$ and $e_{2}$, and $\phi$ be an embedding of $G$ such that $\phi\left(e_{1} \cup e_{2}\right)$ bounds a disk internally disjoint from $\phi(G)$. Then $\phi i s$ primitive (resp. knotless) if and only if $\left.\phi\right|_{G-e_{1}}$ is primitive (resp. knotless).

Proof. Suppose that $\phi$ is primitive. Then $\phi\left(G-e_{1}\right)$ is primitive by Theorem 1.4.

Conversely, suppose that $\left.\phi\right|_{G-e_{1}}$ is primitive. By Theorem 1.4, $\left.\phi\right|_{G / e_{1}-e_{2}}$ is primitive since $\phi(G) / \phi\left(e_{1}\right)-\phi\left(e_{2}\right)=\phi\left(G-e_{1}\right) / \phi\left(e_{2}\right)$. Therefore by Lemma $2.4, \phi_{G / e_{1}}$ is primitive since $\phi\left(e_{2}\right)$ bounds a disk internally disjoint from $\phi(G) / \phi\left(e_{1}\right)$. Hence, by Theorem 1.4, $\phi$ is primitive. 


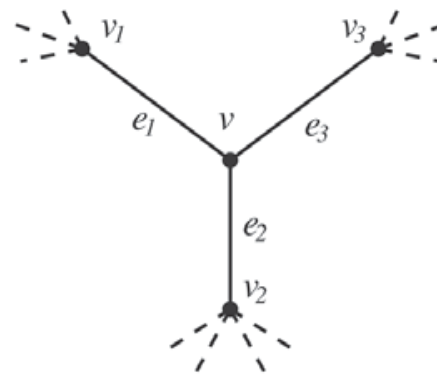

$\mathrm{G}$

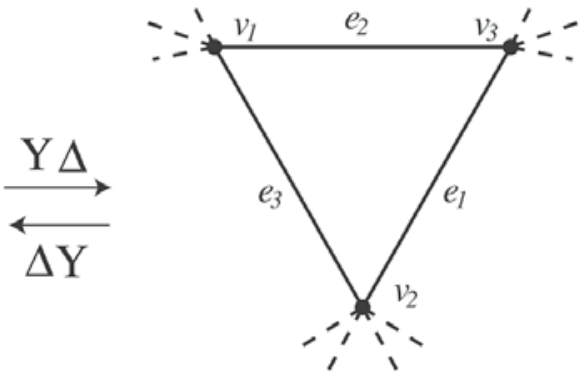

$\mathrm{H}$

Figure $5-Y \Delta$ - and $\Delta Y$-exchange

Let $v$ be a vertex of a graph $G$ of valency three with distinct neighbors $v_{1}, v_{2}, v_{3}$. Let $H$ be obtained from $G$ by deleting $v$ and adding edges $e_{1}, e_{2}, e_{3}$, where $e_{1}=$ $v_{2} v_{3}, e_{2}=v_{3} v_{1}, e_{3}=v_{1} v_{2}$. We say that $H$ is obtained from $G$ by a $Y \Delta$-exchange and that $G$ is obtained from $H$ by a $\Delta Y$-exchange.

Proof (of Theorem 1.5). We use the labelling in figure 5.

Primitive. Suppose that $\phi$ is primitive. Then $\phi^{\prime}\left(H / e_{1}\right)-\phi^{\prime}\left(e_{3}\right)$ is primitive since $\phi^{\prime}\left(H / e_{1}\right)-\phi^{\prime}\left(e_{3}\right)=\phi\left(G /\left(e_{2} \cup e_{3}\right)\right)$. Therefore by Lemma 2.5, $\phi^{\prime}\left(H / e_{1}\right)$ is primitive since $\phi^{\prime}\left(e_{2} \cup e_{3}\right)$ bounds a disk internally disjoint from $\phi^{\prime}\left(H / e_{1}\right)$. And $\phi^{\prime}\left(H-e_{1}\right)$ is primitive since $\phi^{\prime}\left(H-e_{1}\right)=\phi\left(G / e_{1}\right)$. Hence by Theorem $1.4, \phi^{\prime}$ is primitive.

Conversely, suppose that $\phi^{\prime}$ is primitive. Then $\phi\left(G / e_{1}\right)$ is primitive since $\phi\left(G / e_{1}\right)$ $=\phi^{\prime}\left(H-e_{1}\right)$. And $\phi\left(G-e_{1}\right) / \phi\left(e_{2}\right)$ is primitive since $\phi\left(G-e_{1}\right) / \phi\left(e_{2}\right)=\phi^{\prime}(H)-$ $\phi^{\prime}\left(e_{2} \cup e_{3}\right)$. Therefore by Lemma $2.3, \phi\left(G-e_{1}\right)$ is primitive. Hence by Theorem 1.4, $\phi$ is primitive.

Knotless. The proof is similar to above, we need to use only Theorem 1.4, Lemma 2.3, 2.4, 2.5.

\section{Proofs of Theorems on graph minor}

Lemma 3.1. Let $\mathcal{C}$ be a property preserved under taking minors, multiplication of edges, adding loops, and $Y \Delta$-exchanges. Let $H$ be a graph obtained from $G$ by a $\Delta Y$ exchange. Suppose that $G$ does not have the property $\mathcal{C}$ and suppose that $H$ is a forbidden graph for $\mathcal{C}$. Then $G$ is also a forbidden graph for $\mathcal{C}$.

Proof. Let $e$ be an edge of $G$. It is sufficient to show that $G-e$ and $G / e$ have $\mathcal{C}$.

Regarding the triangle of $G$, we have the following three cases: $(\mathrm{A}): e=e_{1},(\mathrm{~B})$ : $\partial e=v_{1} \cup v_{2}$ and $e \neq e_{3}$, and $(\mathrm{C})$ : otherwise. 
(A): In this case, $G-e=H / v_{1} v_{4}$ and thus $G-e$ has $\mathcal{C}$. On the other hand, $G / e$ is obtained from $H /\left\{v_{2} v, v_{3} v\right\}$ by adding a parallel edge to $v_{1} v$. Hence $G / e$ has $\mathcal{C}$. (B): In this case, $G-e$ is obtained from $H-e$ by a $Y \Delta$-exchange. Thus, $G-e$ has $\mathcal{C}$. On the other hand, $G / e$ is obtained from $H /\left\{v_{1} v, v_{2} v\right\}$ by adding a loop to $v$. Hence $G / e$ has $\mathcal{C}$. (C): In this case, $G-e$ and $G / e$ is obtained from $H-e$ and $H / e$ by a $Y \Delta$-exchange. Thus, each of $G-e$ and $G / e$ has $\mathcal{C}$.

This completes the proof.

By a similar argument, we have the following:

Exercise 3.2. Let $\mathcal{C}$ be a property preserved under taking minors, multiplication of edges, and $\Delta Y$-exchanges. Let $H$ be a graph obtained from $G$ by a $Y \Delta$-exchange. Suppose that $G$ does not have $\mathcal{C}$ and suppose that $H$ is a forbidden graph for $\mathcal{C}$. Then $G$ is also a forbidden graph for $\mathcal{C}$.

Proof (of Theorem 1.6). This follows the proof of Theorem 1.4.

Before proving Theorem 1.7, we prove Theorem 1.9.

Proof (of Theorem 1.9). We construct a primitive embedding of a planar graph joined with two vertices as follows. First, we embed a planar graph $G_{0}$ into the 2-sphere $S^{2}$ in $S^{3}$ so that all loops of $G_{0}$ bound open disks in $S^{2}$ disjoint from the image of $G_{0}$, and that each multi edges of $G_{0}$ are mutually parallel in $S^{2}$. The 2-sphere $S^{2}$ separates $S^{3}$ into two 3-balls $B^{+}$and $B^{-}$. Let $v^{+}$and $v^{-}$be vertices contained in int $B^{+}$and int $B^{-}$ respectively. Next we join $v^{+}$and $v^{-}$to each of vertices of $G_{0}$ by monotone edges in $B^{+}$and $B^{-}$respectively. Then we obtain an embedding $\phi$ of a planar graph $G_{0}$ joined with two vertices $v^{+}$and $v^{-}$, say $G$, and prove that this embedding $\phi$ is primitive.

If $G_{0}$ has a loop, then its image under $\phi$ bounds an open disk in $S^{2}$ disjoint from $G_{0}$ by our construction, and hence we may assume that $G_{0}$ has no loops by Lemma 2.4. If $G_{0}$ has multi edges, then the image of them under $\phi$ are mutually parallel in $S^{2}$ by our construction, and hence we may assume that $G_{0}$ has no multi edges by Lemma 2.5 .

The proof is done by induction on the number of edges of $G_{0}$. When $G_{0}$ has no edges, $G_{0}$ is a disjoint union of vertices and $\phi\left(G_{0}\right) *\left(v^{+}, v^{-}\right)$is primitive since it is a trivial theta-curve. Next, let $e$ be an edge of $G_{0}$. Then, $\phi(G-e)$ is primitive by the hypothesis of induction. We shall show that $\phi(G / e)$ is also primitive. Note that $\phi(G / e)$ forms $\phi\left(G_{0} / e\right) *\left(v^{+}, v^{-}\right)$with multi edges $e_{1}^{+}, e_{2}^{+}, e_{1}^{-}, e_{2}^{-}$such that $\partial e_{i}^{ \pm}=(\phi(e) / \phi(e)) \cup v^{ \pm}, e_{1}^{ \pm} \cup e_{2}^{ \pm}$bounds a disk, whose interior is disjoint from $\phi(G / e)$, coming from the triangle defined by $e$ and $v^{ \pm}$. By the hypothesis of induction, $\phi\left(G_{0} / e\right) *\left(v^{+}, v^{-}\right)$is primitive, and by Lemma $2.5, \phi(G / e)$ is primitive.

The genealogies of the $K_{7}$-family and $K_{3,3,1,1}$-family are illustrated in figures 6 and 8 , where " $\rightarrow$ " means a $\Delta Y$-exchange, and all elements of them are in [10] and [9] respectively. 


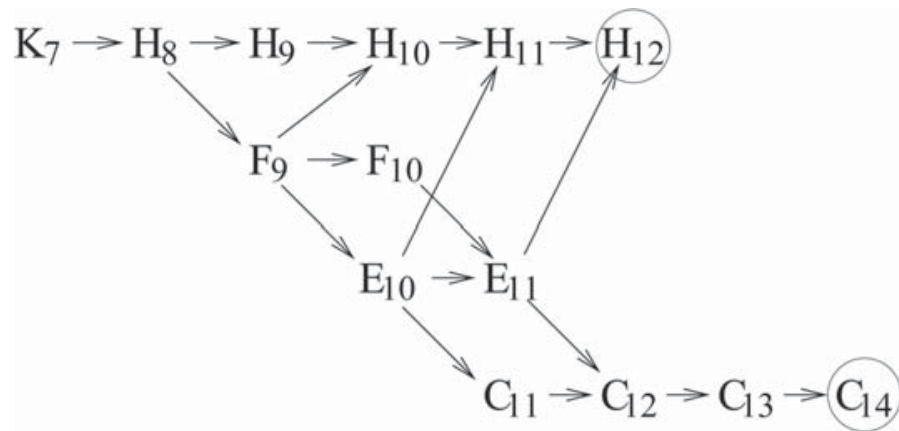

Figure $6-K_{7}$-family

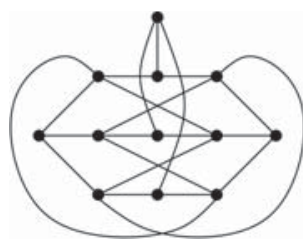

(a) $H_{12}$

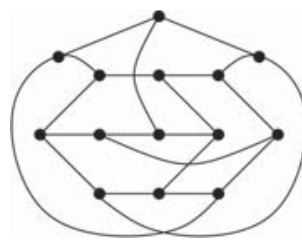

(b) $C_{14}$

Figure $7-H_{12}$ and $C_{14}$ in the $K_{7}$-family

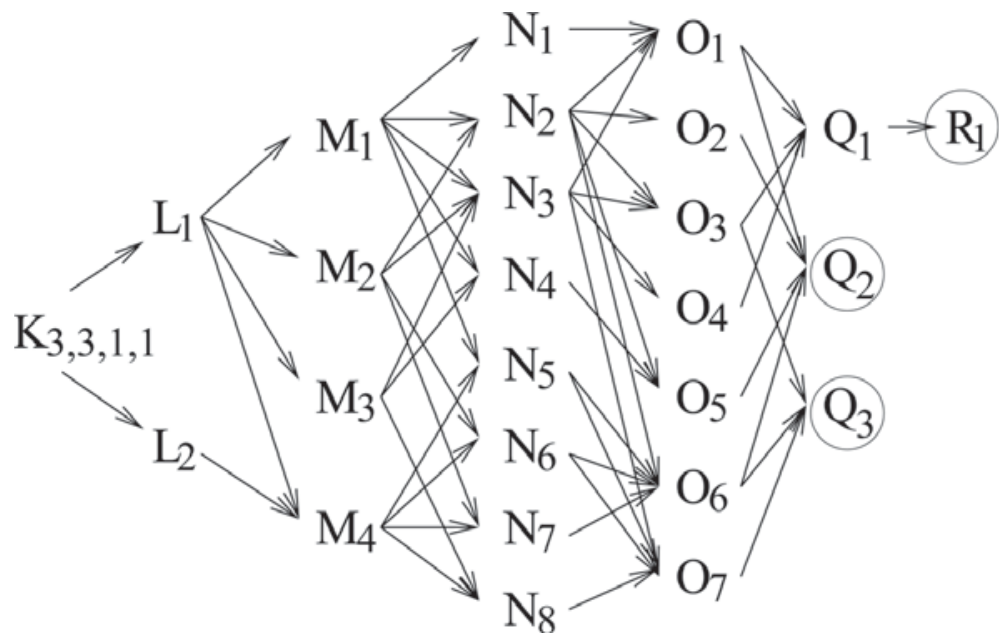

Figure $8-K_{3,3,1,1}$-family 


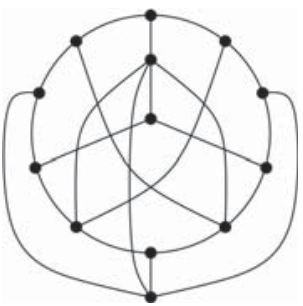

(a) $Q_{2}$

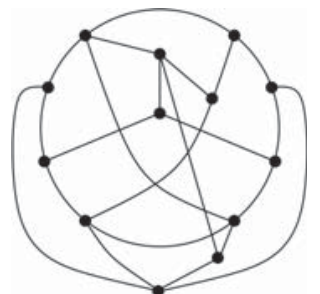

(b) $Q_{3}$

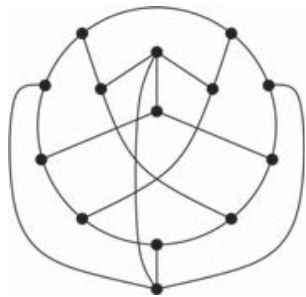

(c) $R_{1}$

Figure $9-Q_{2}, Q_{3}$, and $R_{1}$ in the $K_{3,3,1,1}$-family

Proof (of Theorem 1.7). We review that $K_{7}$ is intrinsically knotted by ConwayGordon ([2]) and $K_{3,3,1,1}$ is also intrinsically knotted by Foisy ([3]). Moreover, by Kohara-Suzuki ([10]), the graphs obtained from $K_{7}$ or $K_{3,3,1,1}$ by $\Delta Y$-exchanges are intrinsically knotted. Thus, $K_{7}$-family and $K_{3,3,1,1}$-family are not primitive by Theorem 1.1.

Next, we show that the "terminal" graphs $H_{12}$ and $C_{14}$ in $K_{7}$-family and $Q_{2}, Q_{3}$, and $R_{1}$ in $K_{3,3,1,1}$-family are forbidden graphs for $\mathcal{P}$. Here, the "terminal" graph means that it can be obtained from $K_{7}$ or $K_{3,3,1,1}$ by $\Delta Y$-exchanges and does not contain 3 -cycles. Thus, $K_{7}$-family and $K_{3,3,1,1}$-family are obtained from these terminal graphs by $Y \Delta$-exchanges. Let $G$ be one of these terminal graphs. It can be checked that for any edge $e$ of $G, G-e$ and $G / e$ are planar graphs joined with two vertices. By Theorem 1.9, $G-e$ and $G$ /e are primitive, hence $G$ is a forbidden graph for $\mathcal{P}$.

We note that $\mathcal{P}$ is preserved under taking minors, multiplication of edges, adding loops, and $Y \Delta$-exchanges. Now, by Lemma 3.1, all graphs in $K_{7}$-family and $K_{3,3,1,1^{-}}$ family are forbidden graphs for $\mathcal{P}$.

\section{Proofs of Theorems on primitive embeddings}

Proof (of Theorem 1.11). Let $G$ be a graph without disjoint cycles and $\phi$ a primitive embedding of $G$. Then by Theorem 1.1, for any connected subgraph $H$ of $G, \phi(H)$ is free. It is sufficient to show that for any disconnected subgraph $H=H_{1} \cup H_{2} \cup$ $\cdots \cup H_{n}, \phi(H)$ is also free. Suppose that $H_{1}$ contains at least one cycle. Then other connected subgraphs $H_{2}, \ldots, H_{n}$ do not contain cycles, so these are trees. Therefore, $\pi_{1}\left(S^{3}-\phi(H)\right) \cong \pi_{1}\left(\phi\left(S^{3}\right)-\phi\left(H_{1}\right)\right)$, hence $\phi(H)$ is free.

Proof (of Theorem 1.12). Put $H_{n}=C_{1} \cup e_{1} \cup \cdots \cup e_{n} \cup C_{2}$, where $C_{1}$ and $C_{2}$ are cycles and $e_{i}$ is an edge connecting $C_{1}$ and $C_{2}$ for $i=1, \ldots, n$.

(i) Let $\phi$ be a primitive embedding of $H_{1}$. Then by contracting $\phi\left(e_{1}\right)$, we have a primitive embedding $\phi\left(H_{1}\right) / \phi\left(e_{1}\right)$ by Theorem 1.4. Since $H_{1} / e_{1}$ does not contain 


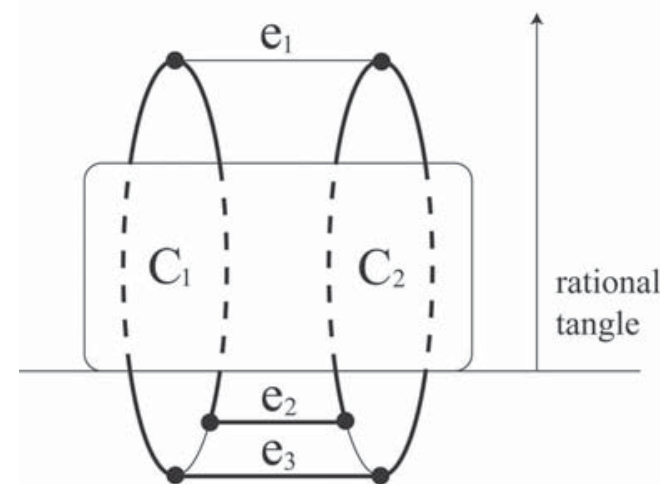

Figure 10 - knotted cycle of $H_{3}$

disjoint cycles, by Theorem 1.11, $\phi\left(H_{1}\right) / \phi\left(e_{1}\right)$ is flat. Hence $\phi\left(H_{1}\right) / \phi\left(e_{1}\right)$ is planar ([18]). By decontracting $e_{1}$, we obtain a primitive embedding $\phi\left(H_{1}\right)$ as a 2-bridge link with an upper tunnel. Finally, it is necessary to check that such an embedding is primitive. By Theorem 1.1, it is sufficient to show that any connected subgraph is free, and it is easy to see.

(ii) Let $\phi$ be a primitive embedding of $H_{2}$. Then by contracting $\phi\left(e_{1}\right)$, we have a primitive embedding $\phi\left(H_{2}\right) / \phi\left(e_{1}\right)$. By Theorem 1.11, $\phi\left(H_{2}\right) / \phi\left(e_{1}\right)$ is flat since $H_{2} / e_{1}$ does not contain disjoint cycles. Hence $\phi\left(H_{2}\right) / \phi\left(e_{1}\right)$ is planar. By decontracting $e_{1}$, we obtain a primitive embedding $\phi\left(H_{2}\right)$ as a 2-bridge link with an upper tunnel and a lower tunnel. Finally, it is necessary to check that such an embedding is primitive. By the similar way, it is easy to see.

(iii) Let $\phi$ be a primitive embedding of $H_{3}$. Then by contracting $\phi\left(e_{1}\right)$, we have a primitive embedding $\phi\left(H_{3}\right) / \phi\left(e_{1}\right)$. By Theorem 1.11, $\phi\left(H_{3}\right) / \phi\left(e_{1}\right)$ is flat since $H_{3} / e_{1}$ does not contain disjoint cycles. Hence $\phi\left(H_{3}\right) / \phi\left(e_{1}\right)$ is planar. By decontracting $e_{1}$, we obtain a primitive embedding $\phi\left(H_{3}\right)$ as a 2-bridge link with an upper tunnel and two parallel lower tunnel. But this embedding is not primitive because it may contain a non-trivial knot consisting of $e_{2}, e_{3}$, the paths of length two in $C_{1}$ and $C_{2}$ (see figure 10).

A 2-string trivial tangle is usually called a rational tangle since it can be represented by a rational number in a standard way. It is necessary and sufficient for the cycle to be unknotted that the continued fraction representation of the upper rational tangle is integral. Thus, the 2-bridge link is a $(2, q)$-torus link and we obtain the desired form. Finally, it is necessary to check that such an embedding is primitive. By the similar way, it is easy to see.

For any rational tangle $(B, T)$ with fraction $r$, there exists an orientation pre- 


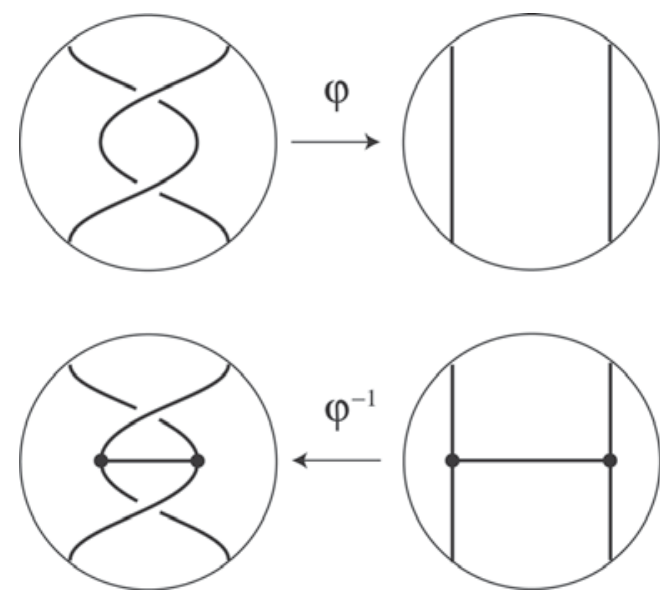

Figure 11 - a rational $H$-shaped tangle with fraction $1 / 2$

serving homeomorphism of pairs $\phi:(B, T) \rightarrow\left(D^{2} \times I,\{x, y\} \times I\right)$. We call the tangle $\phi^{-1}\left(\left(D^{2} \times I, H\right)\right)$ a rational $H$-shaped tangle with fraction $r$, where the tangle $\left(D^{2} \times I, H\right)$ forms a trivial tangle with an edge. See figure 11 .

Proof (of Lemma 1.13). Let $\phi$ be a primitive embedding of $K_{5}^{\prime}$. By Theorem 1.4, $\phi\left(K_{5}^{\prime}\right) / \phi(e)$ is also primitive, and by Theorem 1.11 and $[16,(1.7)]$, primitive embeddings of $K_{5}^{\prime} / e \simeq K_{5}$ are unique up to reflection. Hence, by de-contracting $e$, we obtain a candidate for primitive embeddings of $K_{5}^{\prime}$, where the neighborhood of $\phi(e)$ forms a rational $H$-shaped tangle, that is, a rational tangle with an additional trivial edge $e$. In a rational $H$-shaped tangle, there exists a properly embedded disk that entirely contains the $H$-shaped part. See figure 12 .

By Theorem 1.1, the cycle $v_{1} v_{3} v_{4} v_{2} v_{6} v_{5} v_{1}$ must be a trivial knot. This implies that the rational tangle has a slope $1 / 2 n$ for some integer $n$. Moreover, the cycle $v_{1} v_{3} v_{6} v_{2} v_{4} v_{5} v_{1}$ must be also trivial, it follows that $n=0$ or $n=-1$. Thus, we conclude that all primitive embeddings of $K_{5}^{\prime}$ have only two candidates $(a)$ and $(b)$ in figure 3 corresponding to $n=0$ and $n=-1$ respectively. Conversely, by Theorem 1.9, these two embeddings are primitive since they form into a plane graph joined with the North pole and the South pole by adding some vertices if necessary.

Proof (of Theorem 1.15). Let $G$ be a graph in the Petersen family, $\phi$ a primitive embedding of $G$, and $C_{1} \cup C_{2}$ be a disjoint cycle in $G$. Then $C_{1} \cup C_{2}$ is contained in a subgraph $H$ of $G$ which has a minor $K_{5}^{\prime}$. Since $\phi(H)$ is primitive, its spatial minor $\phi\left(K_{5}^{\prime}\right)$ is also primitive. Hence by Lemma 1.13, $\phi\left(C_{1} \cup C_{2}\right)$ is the trivial link or the Hopf link.

Proof (of Theorem 1.16). Let $\phi$ be a primitive embedding of a graph $G$, and $C=C_{1} \cup \cdots \cup C_{n}$ a disjoint union of $n$-cycles of $G$. First, we contract a path $P_{i}$ of max- 


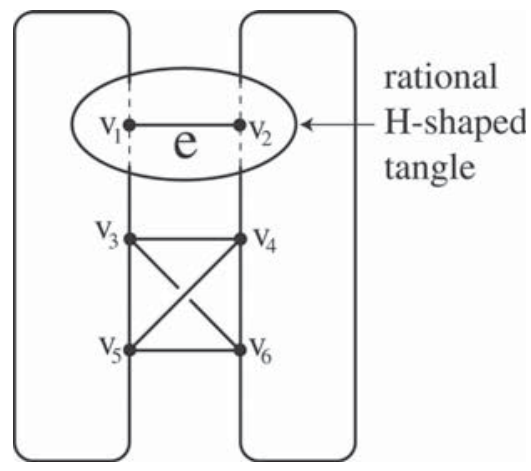

Figure 12 - a candidate for primitive embeddings of $K_{5}^{\prime}$

imal length in each cycle $C_{i}$, and obtain a primitive embedding $\phi(G) / \phi\left(P_{1} \cup \cdots \cup P_{n}\right)$ by the proof of Theorem 1.6. We note that by this contraction, the link type of $\phi(C)$ does not change, thus $\phi(C)$ is equivalent to $\phi\left(C^{\prime}\right)=\phi\left(C_{1}^{\prime}\right) \cup \cdots \cup \phi\left(C_{n}^{\prime}\right)$, where $\phi\left(C_{i}^{\prime}\right)=\phi\left(C_{i}\right) / \phi\left(P_{i}\right)$. Second, we take a spanning tree $T^{\prime}$ of the resultant graph $G^{\prime}=G /\left(P_{1} \cup \cdots \cup P_{n}\right)$ and contract $\phi\left(T^{\prime}\right)$. Then we obtain a trivial bouquet $\phi\left(G^{\prime}\right) / \phi\left(T^{\prime}\right)$ by the primitivity of $\phi$. In other words, the tangle $\left(B, \phi\left(C^{\prime}\right) \cap B\right)$ is a trivial $n$-string tangle, where $B=S^{3}-\operatorname{int} N\left(\phi\left(T^{\prime}\right)\right)$. On the other side, the tangle $\left(N\left(\phi\left(T^{\prime}\right)\right), \phi\left(C^{\prime}\right) \cap N\left(\phi\left(T^{\prime}\right)\right)\right)$ is also trivial, hence the link $\phi\left(C^{\prime}\right)$ is an $n$-bridge link. As we noted above, the link $\phi(C)$ is also $n$-bridge.

Proof (of Theorem 1.18). Suppose that a planar graph $G$ has no disjoint cycles. Then by Theorem 1.11, any primitive embedding of $G$ is also flat, and by [18], it is planar. Therefore $G$ has a unique primitive embedding.

Conversely, suppose that $G$ has disjoint cycles. Since $G$ is planar, there exists a pair of disjoint facial cycles $C_{1}, C_{2}$. Thus $G$ can be embedded in an annulus $A$ so that $C_{1} \cup C_{2}=\partial A$. Let $f_{n}: A \rightarrow S^{3}$ be an embedding such that $f(\partial A)$ forms a $(2,2 n)$-torus link. Then every non-trivial two-component constituent link of $f_{n}(G)$ also forms a $(2,2 n)$-torus link. In particular, $f_{n}\left(C_{1} \cup C_{2}\right)$ is a $(2,2 n)$-torus link.

Claim. $f_{n}$ is a primitive embedding of $G$ for all $n$.

Proof. Let $T$ be a spanning tree for $G$. Then there exists a path $P$ in $T$ which connects $C_{1}$ and $C_{2}$. By contracting $f_{n}(P)$, we have an embedding $f_{n}(G) / f_{n}(P)$ contained in $f_{n}(A) / f_{n}(P)$, which is an immersed disk. Then the "twists" of $f_{n}(A)$ can be untied at the point $f_{n}(P) / f_{n}(P)$. Thus $f_{n}(G) / f_{n}(P)$ is planar, and then by contracting a rest of edges of $f_{n}(T)$, we have a trivial bouquet. Hence $f_{n}$ is primitive for all $n$.

Hence $G$ has infinitely many primitive embeddings. 


\section{References}

[1] C. C. Adams and A. W. Reid, Unknotting tunnels in two-bridge knot and link complements, Comment. Math. Helv. 71 (1996), no. 4, 617-627.

[2] J. H. Conway and C. McA. Gordon, Knots and links in spatial graphs, J. Graph Theory 7 (1983), no. 4, 445-453.

[3] J. Foisy, Intrinsically knotted graphs, J. Graph Theory 39 (2002), no. 3, 178-187.

[4] , A newly recognized intrinsically knotted graph, J. Graph Theory 43 (2003), no. 3, 199-209.

[5] C. McA. Gordon, On primitive sets of loops in the boundary of a handlebody, Topology Appl. 27 (1987), no. 3, 285-299.

[6] J. Hempel, 3-Manifolds, Ann. of Math. Studies, vol. 86, Princeton University Press, Princeton, N. J., 1976.

[7] W. Jaco, Lectures on three-manifold topology, CBMS Regional Conference Series in Mathematics, vol. 43, American Mathematical Society, Providence, R.I., 1980.

[8] T. Kobayashi, Classification of unknotting tunnels for two bridge knots, Proceedings of the Kirbyfest (Berkeley, CA, 1998), Geom. Topol. Monogr., vol. 2, Geom. Topol. Publ., Coventry, 1999, pp. 259-290.

[9] T. Kohara, Knots and links in spatial graphs, Master Thesis, Waseda University.

[10] T. Kohara and S. Suzuki, Some remarks on knots and links in spatial graphs, Knots 90 (Osaka, 1990), de Gruyter, Berlin, 1992, pp. 435-445.

[11] M. Ozawa and Y. Tsutsumi, Minimally knotted spatial graphs are totally knotted, Tokyo J. Math. 26 (2003), no. 2, 413-421.

[12] N. Robertson and P. Seymour, Graph minors, XVI: Wagner's conjecture, to appear.

[13] N. Robertson, P. Seymour, and R. Thomas, Linkless embeddings of graphs in 3-space, Bull. Amer. Math. Soc. (N.S.) 28 (1993), no. 1, 84-89.

[14] _ Kuratowski chains, J. Combin. Theory Ser. B 64 (1995), no. 2, 127-154.

[15] _ _ Petersen family minors, J. Combin. Theory Ser. B 64 (1995), no. 2, 155-184.

[16] $\ldots$, Sachs' linkless embedding conjecture, J. Combin. Theory Ser. B 64 (1995), no. 2, $185-227$.

[17] D. Rolfsen, Knots and links, Mathematics Lecture Series, vol. 7, Publish or Perish Inc., Berkeley, Calif., 1976.

[18] M. Scharlemann and A. Thompson, Detecting unknotted graphs in 3-space, J. Differential Geom. 34 (1991), no. 2, 539-560.

[19] K. Taniyama, Irreducibility of spatial graphs, J. Knot Theory Ramifications 11 (2002), no. 1, $121-124$. 